\title{
Longitudinal Effects of Metabolic Syndrome on Alzheimer and Vascular Related Brain Pathology
}

\author{
Feng Lin ${ }^{\text {a, }}$ Raymond Y. Lo ${ }^{i}$ Daniel Cole Simon Ducharme $^{f-h}$ \\ Ding-Geng Chen ${ }^{b, d}$ Mark Mapstone $^{c}$ Anton Porsteinsson ${ }^{a}$ \\ for the Alzheimer's Disease Neuroimaging Initiative
}

Departments of a Psychiatry, ${ }^{b}$ Biostatistics and Computational Science and ${ }^{\mathrm{C}}$ Neurology, School of Medicine and Dentistry, ${ }^{\mathrm{d}}$ School of Nursing, and ${ }^{\mathrm{e}}$ Department of Brain and Cognitive Science, University of Rochester, Rochester, N.Y., ${ }^{\mathrm{f}}$ Department of Neurology and Psychiatry, Harvard University, Cambridge, Mass., and 9 McLean Hospital, Belmont, Mass., USA; hehavioral Neurology and Neuropsychiatry, Montreal General Hospital, Montreal, Que., Canada; 'Department of Neurology, Buddhist Tzu Chi General Hospital, Tzu Chi University, Hualien, Taiwan, ROC

\section{Key Words}

Metabolic syndrome $\cdot$ Alzheimer pathology $\cdot$ Mild cognitive impairment · Vascular pathology

\section{Abstract}

Background/Aims: This study examines the longitudinal effect of metabolic syndrome (MetS) on brain-aging indices among cognitively normal $(\mathrm{CN})$ and amnestic mild cognitive impairment (aMCI) groups [single-domain aMCI (saMCI) and multiple-domain aMCI (maMCI)]. Methods: The study population included 739 participants $(C N=226$, saMCI $=275$, and maMCI $=238)$ from the Alzheimer's Disease Neuroimaging Initiative, a clinic-based, multicenter prospective cohort. Confirmatory factor analysis was employed to determine a MetS latent composite score using baseline data of vascular risk factors. We examined the changes of two Alzheimer's disease (AD) biomarkers, namely [ $\left.{ }^{18} \mathrm{~F}\right]$ fluorodeoxyglucose (FDG)-positron emission tomography (PET) regions of interest and medial temporal lobe volume over 5 years.

F.L. and R.Y.L. contributed equally to this work. Data used in preparation of this article were obtained from the Alzheimer's Disease Neuroimaging Initiative (ADNI) database (adni.loni.usc.edu). As such, the investigators within the ADNI contributed to the design and implementation of ADNI and/or provided data but did not participate in the analysis or writing of this report. A complete listing of ADNI investigators can be found at: http://adni.loni.usc.edu/wp-content/uploads/how_to_apply/ADNI_Acknowledgement_List.pdf. 
Lin et al.: Longitudinal Effects of Metabolic Syndrome on Alzheimer and Vascular Related Brain Pathology

A cerebrovascular aging index, cerebral white matter (cWM) volume, was examined as a comparison. Results: The vascular risk was similar in all groups. Applying generalized estimating equation modeling, all brain-aging indices declined significantly over time. Higher MetS scores were associated with a faster decline of $\mathrm{CWM}$ in the $\mathrm{CN}$ and maMCI groups but with a slower decrement of regional glucose metabolism in FDG-PET in the saMCI and maMCI groups. Conclusion: At the very early stage of cognitive decline, the vascular burden such as MetS may be in parallel with or independent of $A D$ pathology in contributing to cognitive impairment in terms of accelerating the disclosure of AD pathology.

(C) 2014 S. Karger AG, Base

\section{Introduction}

Over 40 million Americans aged 60 years or older have one or more risk factors for vascular disease, such as obesity, hypertension, and diabetes mellitus [1]. These risk factors contribute to the cognitive decline and development of Alzheimer's disease (AD) [2]. Cardiovascular risk factors are associated with both brain AD pathology (e.g., decreased cortical thickness in the hippocampus and accumulated $\beta$-amyloid deposition in the cerebral cortex) and vascular pathology (e.g., reduced white matter integrity) in subjects across a range of cognitive performance [3-5]. These relationships may be related to the dysregulation of the cerebral blood flow, blood-brain barrier exchange, immune surveillance, trophic support, and hemostatic balance, which ultimately leads to cerebral hypoperfusion, gray matter atrophy, and white matter damage [6]. When vascular risk and AD neuropathology co-occur, $\beta$-amyloid plaques may accumulate in the cerebral vessels and disrupt the vascular integrity, which in turn impair the clearance of $\beta$-amyloid and synergistically accelerate brain damage and cognitive decline [6].

Coexisting cardiovascular risk factors appear to confer greater risks for cognitive decline [7] in addition to AD pathology in aging; however, the relationship between vascular injury and $\mathrm{AD}$ pathology remains unclear. A recent cross-sectional study with a mixed sample of cognitively normal (CN) and moderately demented elderly subjects suggested an association between a higher vascular burden indexed by the Framingham Cardiovascular Risk Profile (FCRP) score and a lower cerebral glucose metabolism restricted to the frontal lobe [8]. In our previous study, higher FCRP scores did not predict greater AD-like pathological progression over time in $\mathrm{CN}$ subjects, subjects with amnestic mild cognitive impairment (aMCI) or early AD [9]. However, whether the summary scores for predicting cardiovascular events is also applicable or sensitive enough to capture AD-like pathology in aging awaits further validation.

Metabolic syndrome (MetS) is a clinical syndrome representing a common combination of several vascular risk factors [obesity, hyperglycemia, high lipid profile, and high blood pressure (BP)]. A growing body of literature suggests that MetS contributes to cognitive decline and predicts $\mathrm{AD}$ prognosis [10]. The present study examined the relationships between MetS and longitudinal changes of two brain-aging indices that reflect early AD pathology, $\left[{ }^{18} \mathrm{~F}\right]$ fluorodeoxyglucose (FDG)-positron emission tomography (PET) in five metaregions (left/right angular gyrus, left/right temporal gyrus, and bilateral posterior cingulated gyrus) and hippocampal volume in $\mathrm{CN}$ subjects (normal aging), single-domain aMCI (saMCI) and multiple-domain aMCI (maMCI) [11]. As a comparison, we also examined the effect of MetS on cerebral white matter (cWM) volume, which is often indicative of cerebrovascular health. We conducted a confirmatory factor analysis (CFA) to determine whether a single latent MetS factor can summarize the pathomechanism across multiple vascular risk factors [12], as a way to quantify the severity of MetS. 


\section{Methods}

\section{Study Population}

Data used in the preparation of this article were obtained from the Alzheimer's Disease Neuroimaging Initiative (ADNI) database (adni.loni.usc.edu). The primary goal of ADNI has been to test whether serial MRI, PET, other biological markers, and clinical and neuropsychological assessment can be combined to measure the progression of $\mathrm{MCI}$ and early AD. The principal investigator of this initiative is Michael W. Weiner, MD. ADNI is the result of the efforts of many co-investigators from a broad range of academic institutions and private corporations, and subjects have been recruited from over 50 sites across the USA and Canada. The initial goal of ADNI was to recruit 800 subjects but ADNI has been followed by ADNI-GO and ADNI-2. To date, these three protocols have recruited over 1,500 adults aged 55-90 years to participate in the research, consisting of $\mathrm{CN}$ elderly individuals, subjects with early or late $\mathrm{MCI}$, and subjects with early AD. Subjects originally recruited for ADNI-1 and ADNI-GO had the option to be followed in ADNI-2. The study procedures were approved by the institutional review boards of all participating institutions. Written informed consent was obtained from all research participants or their representatives. For up-to-date information, see www.adniinfo.org.

Other inclusion criteria included having a minimum of 6 years of education, being fluent in Spanish or English, and having an informant available for participant functional evaluation. Individuals were excluded from the study if they had a Hachinski Ischemic Scale score of 4 or greater and used certain psychoactive medications.

\section{Participants and Timeline}

The present study used data as of July 2013 from ADNI. We included 739 ADNI participants who had information on vascular risk factors for developing MetS composite scores. Nearly all participants had baseline MRI scans but not necessarily for PET scans and cerebrospinal fluid studies, and their length of follow-up varied. The number of participants available for an imaging study or neuropsychological assessment at baseline, 6, 12, 24, 36, 48, and 60 months are presented in the online supplementary Appendix table 1 (for all online suppl. material, see www.karger.com/doi/10.1159/000363285). In the present study, we further categorized the MCI group from the ADNI dataset to the saMCI and maMCI group based on whether the participant had executive deficits (online suppl. Appendix table 2).

\section{MetS Composite Score}

Six vascular risk factors including body mass index (BMI), glucose, triglyceride, total cholesterol, and systolic and diastolic BP at baseline were collected for developing MetS composite scores. Glucose and triglyceride levels were not obtained from fasting blood samples, BMI but not waist circumference was available, and the total cholesterol level instead of high-density lipoprotein cholesterol was available. In the present study, instead of using cutoff scores suggested by the National Cholesterol Education Program Adult Treatment Panel III guidelines [13], we used CFA to develop the composite scores to better quantify MetS (see description in the data analysis section). This method has been used in a previous MetS study [12].

\section{Cognition Index}

Two composite scores for memory and executive function (EF) were developed using factor analyses $[14,15]$. The memory composite score was developed based on the following tests: the memory items from the Mini-Mental State Examination and Alzheimer's Disease Assessment Scale - Cognitive Subscale, the Rey Auditory Verbal Learning Test immediate 
Lin et al.: Longitudinal Effects of Metabolic Syndrome on Alzheimer and Vascular Related Brain Pathology

and delayed scores, and the Logical Memory immediate and delayed scores. The EF composite score was developed based on the Digit Symbol Substitution test, the Wechsler Memory ScaleRevised Digit Span test, the Trail Making Test A and B, category fluency tests (animals and vegetables), and the Clock Drawing Test.

\section{Demographic and Health Variables}

All following data were obtained at baseline: age, sex, education, APOE4 carrier defined as having at least one APOE4 allele (by analyzing blood sample at the National Cell Repository for AD), and presence of cerebral infarction defined as having at least one infarct (using a method developed at the University of California at Davis). $A \beta_{42}$ and phosphorylated tau (p-tau) in cerebrospinal fluid aliquots were measured using the multiplex xMAP Luminex platform (Luminex Corp., Austin, Tex., USA) with immunoassay kit-based reagents (INNO-BIA AlzBio3; Innogenetics, Ghent, Belgium). An $A \beta_{42} / p$-tau ratio was used as the 'AD signature' for which lower $A \beta_{42} / p$-tau ratios indicated an increased burden of AD pathology [16].

\section{Neuroimaging Methods}

Two types of imaging methods were included, namely FDG-PET and structural MRI. The data acquisition protocols for each imaging modality are detailed on the UCLA laboratory of Neuroimaging website (http://www.loni.ucla.edu/ADNI/Data/ADNI_Data.shtml).

\section{FDG-PET}

Methods for PET analysis have been described previously [11]. Briefly, PET images were obtained 30-60 min after injection. Functional volumes were averaged, co-registered to themselves, transformed to a common space, intensity-normalized, and smoothed with an 8-mm FWHM Gaussian kernel. The cerebellar vermis, as defined by the MNI atlas, was used to normalize the intensity of all PET volumes. Predefined meta-regions typically showing altered glucose metabolism within $\mathrm{AD}$ patients were first developed for each participant [11], including the left/right angular gyrus, left/right temporal gyrus, and bilateral posterior cingulated gyrus. A composite score of FDG-PET was then developed by averaging the five metaregions.

\section{Structural MRI}

MRI acquisition and preprocessing have been described in details previously [17]. Briefly, high-resolution T1 structural images were obtained from all participants on either a 1.5T GE scanner (Waukesha, Wisc., USA) or a 1.5T Siemens Medical Solutions MRI (Erlangen, Germany) in adherence with the standardized ADNI protocol. To control for cross-site variation, ADNI has implemented standardized MRI protocols and acquisition parameters, system-specific corrections for gradient nonlinearity and intensity nonuniformity, and phantom-based monitoring of imaging instruments. Additional preprocessing and quality control procedures included gradient warping, scaling, B1 correction, and N3 inhomogeneity correction on all T1-weighted images as performed by the Mayo Clinic.

Cross-sectional image processing was performed using FreeSurfer version 5.1. Each scan is segmented according to an atlas defined by FreeSurfer. This allows for group comparisons at a single time point [18]. Images that did not pass a thorough visual quality control were excluded from the analysis. Variables included in the analyses were cWM volume (left and right hemispheres) and hippocampus volume. Time-dependent total intracranial volume was controlled in all models with structural MRI outcomes. 
Lin et al.: Longitudinal Effects of Metabolic Syndrome on Alzheimer and Vascular Related Brain Pathology

Table 1. Baseline sample characteristics

\begin{tabular}{|c|c|c|c|c|c|}
\hline & \multirow{2}{*}{$\begin{array}{l}\text { Total sample } \\
(n=739)\end{array}$} & \multicolumn{4}{|c|}{ Comparison of three diagnostic groups } \\
\hline & & $\begin{array}{l}\text { CN group } \\
(n=226)\end{array}$ & $\begin{array}{l}\text { saMCI group } \\
(n=275)\end{array}$ & $\begin{array}{l}\text { maMCI group } \\
(\mathrm{n}=238)\end{array}$ & $\begin{array}{l}\text { ANOVA or } \\
\chi^{2} \text { test }\end{array}$ \\
\hline Age, years & $74.51(7.02)$ & $75.86(5.00)^{\mathrm{a}}$ & $72.35(7.87)^{\mathrm{b}}$ & $75.78(7.01)^{\mathrm{a}}$ & $22.32^{* *}$ \\
\hline Education, years & $15.76(2.95)$ & $16.02(2.88)^{\mathrm{a}}$ & $16.33(2.55)^{\mathrm{a}}$ & $14.86(3.23)^{\mathrm{b}}$ & $17.86^{* *}$ \\
\hline APOE4 carrier, n (\%) & $316(42.9 \%)$ & $60(26.5 \%)^{\mathrm{a}}$ & $130(47.4 \%)^{\mathrm{b}}$ & $126(53.2 \%)^{\mathrm{b}}$ & $37.18^{* *}$ \\
\hline Male gender, n (\%) & $433(58.5 \%)$ & $117(51.8 \%)^{\mathrm{a}}$ & $164(59.6 \%)^{a, b}$ & $152(63.9 \%)^{\mathrm{b}}$ & $7.19^{*}$ \\
\hline Presence of cerebral infarction, n (\%) & $61(8.2 \%)$ & $19(8.4 \%)$ & $20(7.4 \%)$ & $22(9.2 \%)$ & 0.59 \\
\hline $\mathrm{A} \beta_{42} / \mathrm{p}$-tau ratio & $9.59(6.85)$ & $10.63(5.45)^{\mathrm{a}}$ & $10.67(7.61)^{a}$ & $7.38(6.39)^{b}$ & $11.01^{* *}$ \\
\hline BMI & $26.67(4.46)$ & $26.74(4.37)$ & $26.75(4.59)$ & $26.51(4.42)$ & 0.23 \\
\hline Glucose $\mathrm{e}^{\ddagger}$ & $4.60(0.19)$ & $4.61(0.18)$ & $4.60(0.19)$ & $4.59(0.19)$ & 0.21 \\
\hline Triglyceride $^{\ddagger}$ & $4.84(0.51)$ & $4.83(0.52)$ & $4.88(0.50)$ & $4.80(0.52)$ & 1.70 \\
\hline Total cholesterol & $195.50(41.32)$ & $192.81(40.02)$ & $199.49(41.69)$ & $193.40(41.93)$ & 2.01 \\
\hline Systolic BP & $134.65(17.28)$ & $134.43(16.81)$ & $133.55(16.51)$ & $136.15(18.51)$ & 1.48 \\
\hline Diastolic BP & $74.84(9.66)$ & $74.63(10.15)$ & $74.88(9.66)$ & $75.00(9.21)$ & 0.09 \\
\hline MetS composite score & $0.0002(0.90)$ & $0.01(0.91)$ & $0.03(0.94)$ & $-0.04(0.83)$ & 0.34 \\
\hline EF & $0.27(0.81)$ & $0.70(0.67)^{\mathrm{a}}$ & $0.66(0.48)^{\mathrm{a}}$ & $-0.59(0.81)^{\mathrm{b}}$ & $426.46^{* *}$ \\
\hline Memory & $0.35(0.35)$ & $0.98(0.53)^{\mathrm{a}}$ & $0.31(0.60)^{\mathrm{b}}$ & $-0.21(0.52)^{c}$ & $436.46^{* *}$ \\
\hline FDG-PET & $6.24(0.67)$ & $6.42(0.62)^{\mathrm{a}}$ & $6.39(0.61)^{\mathrm{a}}$ & $5.89(0.66)^{\mathrm{b}}$ & $29.81^{* *}$ \\
\hline Hippocampus, $\mathrm{cm}^{3}$ & $6.63(1.10)$ & $7.23(0.91)^{\mathrm{a}}$ & $6.87(1.17)^{b}$ & $6.38(1.03)^{c}$ & $32.57^{* *}$ \\
\hline Left-hemisphere cWM, $\mathrm{cm}^{3}$ & $213.39(30.40)$ & $215.46(29.58)$ & $214.55(31.28)$ & $209.83(30.38)$ & 1.76 \\
\hline Right-hemisphere cWM, $\mathrm{cm}^{3}$ & $216.21(30.03)$ & $217.17(29.06)$ & $217.79(31.86)$ & 213.57 (29.37) & 0.99 \\
\hline
\end{tabular}

Unless specifically indicated, all data are represented as mean (SD). ${ }^{\ddagger} \log$-transformed; ${ }^{*} \mathrm{p}<0.05 ;{ }^{* *} \mathrm{p}<0.001$. Each superscript letter denotes a subset of categories whose column proportions do not differ significantly from each other.

\section{Statistical Analyses}

CFA was conducted using Mplus 7.0 to determine the structure of MetS. Model fit indices included a root mean squared error of approximation of $\leq 0.06$, a standardized root mean square residual of $\leq 0.08$, and a comparative fit index of $\geq 0.90$. We followed the model developed in a previous CFA study on MetS, taking BMI as the reference and correlating triglyceride with the total cholesterol [12]. We also correlated systolic with diastolic BP. A composite score of MetS generated from the CFA was used for subsequent analyses. Given the similarity of individual vascular risk factors by diagnostic group (table 1), we only conducted a single CFA using the entire sample.

Other analyses were conducted using SPSS 19.0. A comparison of demographic and health variables by diagnostic group was performed using analysis of variance (ANOVA) for continuous variables and the $\chi^{2}$ test for categorical variables, with Bonferroni as post hoc analysis.

We examined the relationships between MetS and changes of each brain-aging index and cognitive index over time within each group by a series of generalized estimating equations with AR (1) working correlation matrix. All brain-aging indices were normally distributed in each diagnostic group. The linear model therefore chosen was $y=\beta_{0}+\beta_{1} \operatorname{cov}_{1} \ldots \beta_{\mathrm{n}} \operatorname{cov}_{\mathrm{n}}+$ $\beta_{(\mathrm{n}+1)}$ time $+\beta_{(\mathrm{n}+2)}$ MetS $+\beta_{(n+3)}$ time $\times$ MetS $+\varepsilon\left(\operatorname{cov}_{1}\right.$ to $\operatorname{cov}_{\mathrm{n}}=$ potential covariates; $\beta_{(\mathrm{n}+1)}$ $=$ changes of the brain-aging index over time; $\beta_{(\mathrm{n}+2)}=$ the influence of MetS on the baseline level of the brain-aging index; $\beta_{(\mathrm{n}+3)}=$ the influence of MetS on the rate of changes of the brain-aging index over time; $\varepsilon=$ the error term). The $\mathrm{p}$ value for statistical significance was set at 0.05 . 
Lin et al.: Longitudinal Effects of Metabolic Syndrome on Alzheimer and Vascular Related Brain Pathology

Fig. 1. Developing the MetS composite score using CFA. Glucose and triglyceride were log-transformed. All vascular risk factors were standardized. BMI was taken as a reference. Equation for developing MetS composite score: $0.46 \mathrm{BMI}+0.32$ glucose +0.48 triglyceride -0.19 total cholesterol + 0.11 systolic BP. * $\mathrm{p}<0.05$; *** $\mathrm{p}<$ 0.001 .

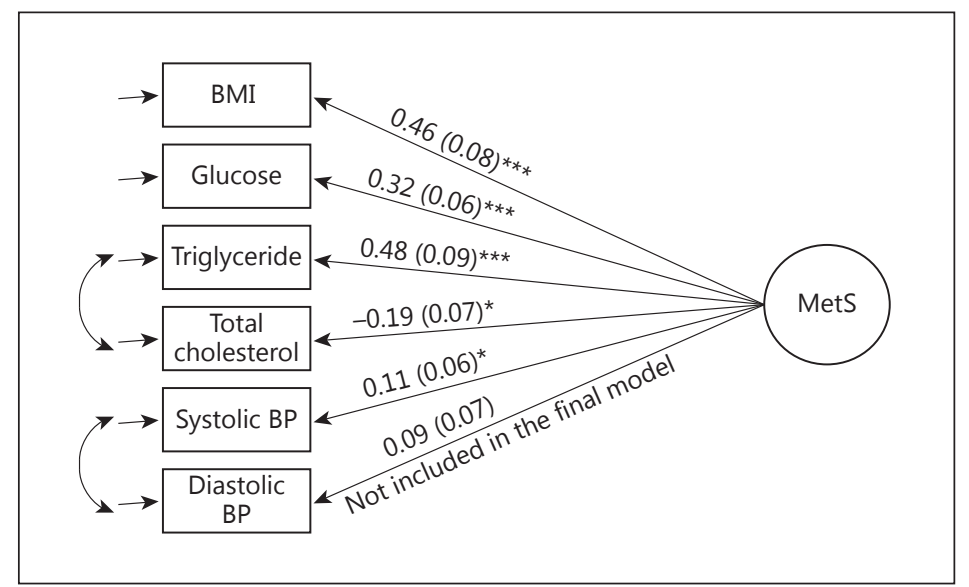

\section{Results}

\section{CFA Analysis for the MetS Composite Score}

CFA was conducted with and without correlations between total cholesterol and triglyceride as well as between systolic and diastolic BP. The best model fit is presented in figure 1 . Model fit indices (root mean squared error of approximation $=0.06$, standardized root mean square residual $=0.04$, and comparative fit index $=0.93$ ) indicated a good model fit. All significant factors were included in the development of the MetS composite score; diastolic BP was excluded due to its insignificant correlation with the latent variable.

\section{Baseline Sample Characteristics}

The saMCI group was significantly younger than the other two groups. The maMCI group had a significantly lower education, and the CN group had fewer APOE4 carriers. The maMCI group had a lower $A \beta_{42} / p$-tau ratio, lower mean EF composite scores, and lower levels of glucose metabolism (FDG-PET) than the other two groups, while three groups significantly differed in their memory scores and hippocampus volumes.

When examining the correlation between $\mathrm{A} \beta_{42} / \mathrm{p}$-tau ratio and MetS within each group, lower $A \beta_{42} / p$-tau ratios were significantly associated with lower MetS scores in the saMCI group $(r=0.24, p=0.002, n=168)$, approaching significance in the maMCI group $(r=0.16$, $\mathrm{p}=0.067, \mathrm{n}=131)$ but remaining nonsignificant in the $\mathrm{CN}$ group $(\mathrm{r}=0.04, \mathrm{p}=0.65, \mathrm{n}=109)$.

\section{Effect of MetS on Brain-Aging and Cognition Indices over Time}

Table 2 shows the association between MetS, brain-aging and cognition indices over time within each group separately. All brain-aging indices as well as memory declined significantly over time in each group; EF significantly declined over time in the saMCI and maMCI groups but not in the CN group $\left(\beta_{(n+1)}\right.$ terms). Higher MetS was associated with a higher hippocampus and left hemisphere cWM volume at baseline in the maMCI group but not associated with any other brain-aging or cognition indices at baseline in the other groups $\left(\beta_{(\mathrm{n}+2)}\right.$ terms). Higher MetS was significantly associated with a faster decline of the left hemisphere cWM volume in the CN and maMCI groups and with a slower decline of FDG-PET in the saMCI and maMCI groups. MetS was not associated with rates of change in the hippocampal volume, right hemisphere $\mathrm{cWM}$, or cognition indices $\left(\beta_{(\mathrm{n}+3)}\right.$ terms). 


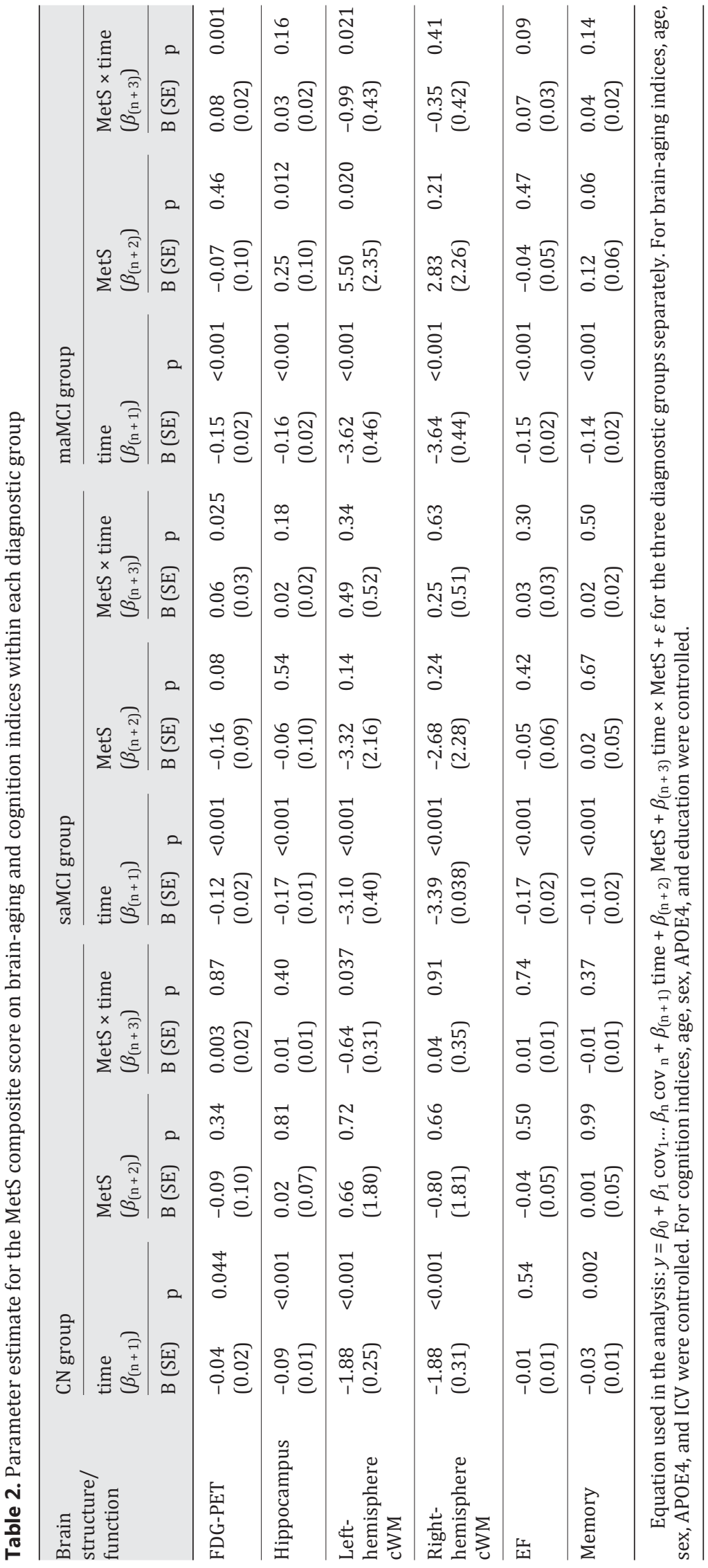




\section{Discussion}

In the present study on three nondementia scenarios ( $\mathrm{CN}$, saMCI, and maMCI), we examined brain-aging indices including AD-like (hypometabolism in angular, temporal, and posterior cingulated gyri as well as hippocampal atrophy) and vascular pathological (reduced cWM volume) changes. All brain-aging indices declined significantly over time across all groups. The MCI groups also had a significant cognitive decline over time. The influence of MetS on the change in the brain-aging indices over time differed across the groups, particularly in the cerebrovascular aspect that higher MetS scores accelerated the decline of left hemisphere cWM in the maMCI and CN groups. We found no evidence, at least in the early stage of cognitive aging, to support the hypothesis that MetS would contribute the AD pathology. The association between greater MetS and a slower decline in FDG in the MCI groups should be cautiously interpreted given the potential inverse correlations found between $\mathrm{AD}$ pathology $\left(\mathrm{A} \beta_{42} / \mathrm{p}\right.$-tau ratio) and MetS levels.

The study has several unique features. First, we developed a MetS composite score to reflect relevant vascular burden. It provided a more direct and quantitative way to capture the severity of MetS than simply using the presence or absence of MetS diagnosis. Of note, given the lack of data on high-density lipoprotein, we used total cholesterol when developing the latent MetS score. Previous studies have failed to find a consistent effect of total cholesterol on brain pathology as opposed to other individual vascular risk factors, suggesting that total cholesterol is a complex if its components are not singled out. Second, we compared three nondementia elderly groups, which may either present a normal aging process, AD pathology, or AD/vascular pathology. Such a comparison provides the possibility to explicate the influence of MetS on different mechanisms involved in the very early stage of cognitive decline. Third, we controlled multiple factors including the genetic (APOE4 allele) background and brain size (intracranial volume) important for confounding the MetS effects but rarely available in longitudinal studies.

Although accumulated literature supports the association between individual vascular risk factors and cerebrovascular pathology [19], there is a lack of evidence regarding the longitudinal effects of MetS on brain aging within the context of AD pathology. In the present study, MetS accelerated the loss of left-, but not right-hemisphere cWM in cognitively healthy elderly adults and those with maMCI. MetS seemed to favorably affect the left hemisphere, and this finding was in line with two previous studies regarding the association between Framingham's vascular index and cerebral glucose hypometabolism [8] as well as the association between hypertension and a lower cerebral blood flow. Both studies preferred the left hemisphere among cognitively healthy elderly adults [20]. The left hemisphere was found to be more sensitive to BP changes [21], and therefore, MetS may have a greater impact on lefthemisphere cWM. MetS may alter the neurovascular unit, leading to the damage of cWM [6]. Higher MetS was associated with more prevalent and severe silent brain infarction [22], lower fractional anisotropy of diffusion tensor imaging (DTI) [23], and higher WM hyperintensity [24]. Previous studies also found that the maMCI group has significantly more WM damage than the saMCI group [25]. When AD pathology outweighs cerebrovascular pathology, as often seen in the saMCI group, AD pathology may dominate the degenerative process, making effects of MetS on saMCI less conspicuous.

AD-like pathological changes including synaptic dysfunction and neuronal death were reflected by regional glucose hypometabolism and hippocampal atrophy, respectively, in our study. Higher MetS was associated with a slower decline in AD-like pathological progression over time in the MCI groups. We also found that higher $A \beta_{42} / p$-tau ratios, indicating lower $A D$ pathology, were associated with higher MetS levels. Similar associations were found in previous studies. For example, a higher BMI was associated with lower levels of neuritic 
plaques, diffuse plaques, and neurofibrillary tangles in the brain [26]. Having diabetes mellitus was associated with less severe $\beta$-amyloid accumulation in the hippocampus [27]. These findings may at first sight contradict to our concept that increased vascular burden is associated with higher risks of AD. One possibility is that MCI participants were enrolled based on cognitive function, and at a given level of cognitive impairment, subjects with an increased MetS burden may have less severe AD pathology or less AD-like pathological progression. Such proposition is based on the evidence that microvascular disease may decrease the threshold for AD pathology or amplify the consequence of neurodegenerative pathology to produce clinical symptoms such as memory or executive deficits $[19,28]$. Higher MetS may not directly protect the brain from $\mathrm{AD}$ pathology but rather accelerate the disclosure of $\mathrm{AD}$ pathology.

There are several limitations in our study. First, the vascular burden in these cohorts was relatively low (Hachinski <4), limiting the generalizability of our findings to subjects with mild degrees of MetS. Next, we used T1-weighted MRI-based cWM volume to reflect WM integrity, and this measurement may be less sensitive than DTI. We expect to have more DTI data from ADNI-GO and ADNI-2 to further validate our current findings. Third, MetS composite scores were determined at baseline, but the status or severity of MetS may fluctuate over time as well, especially for those who had concurrent medical control. Collecting information on time-varying vascular risks is warranted in future investigation. Finally, less than $10 \%$ of the participants had 5 years' follow-up. In our relatively healthy sample, the influence of MetS on AD pathology may not be detectable within this observation period.

The neurovascular hypothesis was proposed within the context of AD such that the interaction occurs later in the disease course with more $\beta$-amyloid deposition or neurofibrillary tangle burden [6], while at the very early stage of cognitive decline, the vascular burden such as MetS may be in parallel with or independent of AD pathology in contributing to cognitive impairment in terms of accelerating the disclosure of AD pathology.

\section{Acknowledgments}

Data collection and sharing for this project was funded by ADNI (National Institutes of Health grant U01 AG024904) and DOD ADNI (Department of Defense award number W81XWH12-2-0012). ADNI is funded by the National Institute on Aging, the National Institute of Biomedical Imaging and Bioengineering, and through generous contributions from the Alzheimer's Association, Alzheimer's Drug Discovery Foundation, BioClinica, Inc., Biogen Idec Inc., Bristol-Myers Squibb Company, Eisai Inc., Elan Pharmaceuticals, Inc., Eli Lilly and Company, F. Hoffmann-La Roche Ltd. and its affiliated company Genentech, Inc., GE Healthcare, Innogenetics, N.V., IXICO Ltd., Janssen, Alzheimer Immunotherapy Research and Development, LLC., Johnson and Johnson Pharmaceutical Research and Development LLC., Medpace, Inc., Merck \& Co., Inc., Meso Scale Diagnostics, LLC., NeuroRx Research, Novartis Pharmaceuticals Corporation, Pfizer Inc., Piramal Imaging, Servier, Synarc Inc., and Takeda Pharmaceutical Company. The Canadian Institutes of Health Research is providing funds to support ADNI clinical sites in Canada. Private sector contributions are facilitated by the Foundation for the National Institutes of Health (www.fnih.org). The grantee organization is the Northern California Institute for Research and Education, and the study is coordinated by the Alzheimer's Disease Cooperative Study at the University of California, San Diego. ADNI data are disseminated by the Laboratory for Neuro Imaging at the University of Southern California.

The manuscript preparation was supported by the University of Rochester CTSA award No. KL2 TR000095 from the National Center for Advancing Translational Sciences of the National Institutes of Health to F.L. The content is solely the responsibility of the authors and does not necessarily represent the official views of the National Institutes of Health. 
Lin et al.: Longitudinal Effects of Metabolic Syndrome on Alzheimer and Vascular Related Brain Pathology

\title{
Disclosure Statement
}

\author{
The authors disclose no conflicts of interest.
}

\section{References}

1 Roger VL, Go AS, Lloyd-Jones DM, et al: Heart disease and stroke statistics - 2011 update: a report from the American Heart Association. Circulation 2011;123:e18-e209.

$\checkmark 2$ Gorelick PB, Scuteri A, Black SE, et al: Vascular contributions to cognitive impairment and dementia: a statement for healthcare professionals from the American Heart Association/American Stroke Association. Stroke 2011;42:2672-2713.

-3 Gazdzinski S, Kornak J, Weiner MW, Meyerhoff DJ: Body mass index and magnetic resonance markers of brain integrity in adults. Ann Neurol 2008;63:652-657.

-4 Rodrigue KM, Rieck JR, Kennedy KM, Devous MD Sr, Diaz-Arrastia R, Park DC: Risk factors for beta-amyloid deposition in healthy aging: vascular and genetic effects. JAMA Neurol 2013;70:600-606.

5 Moran C, Phan TG, Chen J, Blizzard L, Beare R, Venn A, Munch G, Wood AG, Forbes J, Greenaway TM, Pearson $\mathrm{S}$, Srikanth V: Brain atrophy in type 2 diabetes: regional distribution and influence on cognition. Diabetes Care 2013;36:4036-4042.

6 Iadecola C: The overlap between neurodegenerative and vascular factors in the pathogenesis of dementia. Acta Neuropathol 2010;120:287-296.

7 Profenno LA, Porsteinsson AP, Faraone SV: Meta-analysis of Alzheimer's disease risk with obesity, diabetes, and related disorders. Biol Psychiatry 2011;67:505-512.

-8 Kuczynski B, Jagust W, Chui HC, Reed B: An inverse association of cardiovascular risk and frontal lobe glucose metabolism. Neurology 2009;72:738-743.

-9 Lo RY, Jagust WJ: Vascular burden and Alzheimer disease pathologic progression. Neurology 2012;79:13491355.

10 Panza F, Frisardi V, Capurso C, Imbimbo BP, Vendemiale G, Santamato A, D’Onofrio G, Seripa D, Sancarlo D, Pilotto A, Solfrizzi V: Metabolic syndrome and cognitive impairment: current epidemiology and possible underlying mechanisms. J Alzheimers Dis 2010;21:691-724.

-11 Landau SM, Harvey D, Madison CM, Koeppe RA, Reiman EM, Foster NL, Weiner MW, Jagust WJ: Associations between cognitive, functional, and FDG-PET measures of decline in AD and MCI. Neurobiol Aging 2011;32: 1207-1218.

12 Stevenson JE, Wright BR, Boydstun AS: The metabolic syndrome and coronary artery disease: a structural equation modeling approach suggestive of a common underlying pathophysiology. Metabolism 2012;61: 1582-1588.

-13 Expert panel on Detection, Evaluation, and Treatment of High Blood Cholesterol in Adults: Executive summary of the third report of the national cholesterol education program (NCEP) expert panel on detection, evaluation, and treatment of high blood cholesterol in adults (adult treatment panel III). JAMA 2001;285:24862497.

14 Crane PK, Carle A, Gibbons LE, Insel P, Mackin RS, Gross A, Jones RN, Mukherjee S, Curtis SM, Harvey D, Weiner M, Mungas D: Development and assessment of a composite score for memory in the Alzheimer's Disease Neuroimaging Initiative (ADNI). Brain Imaging Behav 2012;6:502-516.

15 Gibbons LE, Carle AC, Mackin RS, Harvey D, Mukherjee S, Insel P, Curtis SM, Mungas D, Crane PK: A composite score for executive functioning, validated in Alzheimer's Disease Neuroimaging Initiative (ADNI) participants with baseline mild cognitive impairment. Brain Imaging Behav 2012;6:517-527.

16 De Meyer G, Shapiro F, Vanderstichele H, Vanmechelen E, Engelborghs S, De Deyn PP, Coart E, Hansson O, Minthon L, Zetterberg H, Blennow K, Shaw L, Trojanowski JQ: Diagnosis-independent Alzheimer disease biomarker signature in cognitively normal elderly people. Arch Neurol 2010;67:949-956.

17 Jack CR Jr, Bernstein MA, Fox NC, et al: The Alzheimer's Disease Neuroimaging Initiative (ADNI): MRI methods. J Magn Reson Imaging 2008;27:685-691.

$\checkmark 18$ Fischl B, Dale AM: Measuring the thickness of the human cerebral cortex from magnetic resonance images. Proc Natl Acad Sci USA 2000;97:11050-11055.

19 Knopman DS, Roberts R: Vascular risk factors: imaging and neuropathologic correlates. J Alzheimers Dis 2010; 20:699-709.

20 Dai W, Lopez OL, Carmichael OT, Becker JT, Kuller LH, Gach HM: Abnormal regional cerebral blood flow in cognitively normal elderly subjects with hypertension. Stroke 2008;39:349-354.

-21 Dicker D, Maya I, Vasilevsky V, Gofman M, Markowitz D, Beilin V, Sarid M, Yosefy C: Blood pressure variability in acute ischemic stroke depends on hemispheric stroke location. Blood Press 2006;15:151-156.

22 Kwon HM, Kim BJ, Park JH, Ryu WS, Kim CK, Lee SH, Ko SB, Nam H, Lee YS, Yoon BW: Significant association of metabolic syndrome with silent brain infarction in elderly people. J Neurol 2009;256:1825-1831.

-23 Shimoji K, Abe O, Uka T, Yasmin H, Kamagata K, Asahi K, Hori M, Nakanishi A, Tamura Y, Watada H, Kawamori R, Aoki S: White matter alteration in metabolic syndrome: diffusion tensor analysis. Diabetes Care 2013;36: 696-700. 
24 Portet F, Brickman AM, Stern Y, Scarmeas N, Muraskin J, Provenzano FA, Berr C, Bonafe A, Artero S, Ritchie K, Akbaraly TN: Metabolic syndrome and localization of white matter hyperintensities in the elderly population. Alzheimers Dement 2012;8:S88-S95. e1.

25 Li H, Liang Y, Chen K, Li X, Shu N, Zhang Z, Wang Y: Different patterns of white matter disruption among amnestic mild cognitive impairment subtypes: relationship with neuropsychological performance. J Alzheimers Dis 2013;36:365-376.

26 Buchman AS, Schneider JA, Wilson RS, Bienias JL, Bennett DA: Body mass index in older persons is associated with Alzheimer disease pathology. Neurology 2006;67:1949-1954.

-27 Beeri MS, Silverman JM, Davis KL, Marin D, Grossman HZ, Schmeidler J, Purohit DP, Perl DP, Davidson M, Mohs RC, Haroutunian V: Type 2 diabetes is negatively associated with Alzheimer's disease neuropathology. J Gerontol A Biol Sci Med Sci 2005;60:471-475.

28 Kalaria RN: Neurodegenerative disease: diabetes, microvascular pathology and Alzheimer disease. Nat Rev Neurol 2009;5:305-306. 